\title{
NON-SINUSOIDAL BENDING WAVES OF SPERM FLAGELLA*
}

\author{
By C. J. BROKAW \\ Division of Biology, California Institute of Technology, \\ Pasadena, California
}

(Received 12 December 1964)

\section{INTRODUCTION}

The analyses of flagellar movement stimulated by Sir James Gray's (r955) photographic study of the movement of sea-urchin spermatozoa have used sine waves as a convenient mathematical model for the active bending waves of flagella. Machin (1958), following a suggestion of Pringle (1957), outlined an attractively simple model for the mechanical co-ordination of bending elements distributed along a flagellum to give propagated sine waves. However, in a later paper, Machin (1963) showed that these bending elements must behave non-linearly, in which case the bending waves will probably not be sinusoidal. Brokaw \& Wright (I963) presented photographs showing that in at least one case-the large posterior flagellum of the dinoflagellate Ceratium - the bending waves are not sine waves, but instead contain regions of constant bending, forming circular arcs, separated by shorter unbent regions.

In this paper photographs of flagellar bending waves of spermatozoa from marine invertebrate representatives of three animal phyla will be presented and discussed with reference to the description of bending waves proposed by Brokaw and Wright. Differences have been observed between the spermatozoa of these three species in respect of their movements under certain experimental conditions, but the common features of their movements will be emphasized in this paper.

\section{METHODS}

The animals used as sources of spermatozoa were collected in Newport Bay, California, and maintained at the Kerckhoff Marine Laboratory for periods up to several weeks before use. Spermatozoa of a sea urchin, Lytechinus pictus, were obtained by injection of $0.5 \mathrm{M}-\mathrm{KCl}$ into the perivisceral cavity to induce spawning (Tyler, 1949). Spermatozoa of a polychaete annelid, Chaetopterus variopedatus, were obtained by cutting one or several parapodia. Spermatozoa of a tunicate, Ciona intestinalis, were obtained by cutting the sperm duct, exposed by dissection of the animal. Concentrated spermatozoa were stored in glass pipettes for up to $2 \mathrm{hr}$. at room temperature $\left(18^{\circ} \mathrm{C}\right.$.). Small amounts of the concentrated spermatozoa were diluted as needed, and allowed to stand for $3 \mathrm{~min}$. at room temperature before a drop was placed on a standard microscope slide and covered with a coverglass. The spermatozoa

- This work has been supported in part by a grant from the United States Public Health Service (RG-6965). 
were diluted with a filtered sea-water solution containing $5 \times 10^{-4} \mathrm{M}$ ethylenediaminetetra-acetate and $0 . \mathrm{I} M$ histidine, to prolong the period of motility in dilute solution (Tyler, 1953), and $\mathrm{r} \%$ polyvinylpyrrolidinone, to reduce the tendency of the spermatozoa to stick to the slide and coverglass. The $\mathrm{pH}$ was adjusted to $8 \cdot \mathrm{I}$ by adding tris-hydroxymethyl aminomethane. This solution had a viscosity of $\mathrm{r} \cdot 4$ centipoise at $16^{\circ} \mathrm{C}$.

Headless spermatozoa of Lytechinus were obtained by repeatedly drawing a diluted sperm suspension in and out of a fine-tipped glass pipette. Best results were obtained when the solution also contained methyl cellulose, to raise its viscosity to approximately 5 centipoise, and $O \cdot I M$ thiourea, to counteract the reduction in amplitude of the flagellar bending waves which normally occurs at this viscosity (Brokaw, 1964). These suspensions were examined immediately, as the headless spermatozoa showed normal motility for only a minute or two. A similar mechanical treatment of Ciona spermatozoa, in the ordinary sea-water solution, caused breakage of the flagella rather than removal of heads. These spermatozoa, with shorter than normal flagella, often remained motile for as long as undamaged spermatozoa.

The microscope stage was cooled by circulation of coolant at $15^{\circ} \mathrm{C}$. The temperature at the point of observation of the spermatozoa was taken to be $16^{\circ} \mathrm{C}$.; although this could not be determined exactly, it should have been constant within a few tenths of a degree throughout the observations.

The electronic flash apparatus described previously (Brokaw, 1963) was used, with only minor modifications, for stroboscopic observations and multiple-flash photography. Photographs were taken on Kodak Tri-X film with a magnification on film of $\times 200$. The flash apparatus is designed to produce flashes lasting less than $0.1 \mathrm{msec}$, in contrast to the 2 msec. flashes used by Gray (1955). Since the flagellum has a diameter of approximately $0 \cdot 2 \mu$ and moves $\mathrm{I}-\mathrm{I} \cdot 5 \mu$ in 2 msec., this decreased flash duration gives significantly better resolution. Improvement of Tri-X film since 1955 has also contributed to increased photographic resolution.

\section{OBSERVATIONS}

\section{General characteristics of the bending waves}

Wave patterns of spermatozoa of Lytechinus, Ciona, and Chaetopterus under 'normal' conditions are illustrated by the photographs in Pl. I, figs. I- -6 . The photographs are multiple-flash exposures in which the initial flash can be identified by its higher intensity. In some photographs the subsequent path of the head is indicated by a streak exposed by the low-intensity constant illumination used for visual observation of the spermatozoa. Photographs of this quality are obtainable only because the flagellar waves are planar, and because the spermatozoa accumulate near the surfaces of the slide and coverglass, with their wave planes parallel to the surfaces (Gray, 1955; Rothschild, 1963). Most of the wave patterns which were photographed are asymmetrical, since spermatozoa with asymmetrical waves swim in circles and can be followed for sufficient time to allow accurate focusing and selection of a region clear of other spermatozoa. The minority of spermatozoa which have effectively symmetrical wave patterns and are swimming in nearly straight lines are more difficult to photograph. The asymmetry appears to involve the amount of bending, rather than the 
length of flagellum which is bent. Very asymmetrical wave patterns were frequently seen, particularly with nearly moribund, headless spermatozoa. These often showed slowly propagating normal bends on one side of the flagellum combined with a complete absence of bending on the other side.

When perfectly focused the image of the sperm flagellum in the photographs has a width equivalent to about $0_{4} 4 \mu$, not more than twice the diameter of the flagellum, and the uncertainty in the position of the flagellum is small. Many photographs have been obtained in which most of the bent region of the flagellum could be matched exactly by a circular arc, as illustrated in the enlarged photograph in Pl. I, fig. 7 . Between the bent regions the flagellum is nearly straight. On the other hand the curvature of lengths of flagellum much shorter than the radius of curvature cannot be measured accurately, so that a detailed examination of the pattern of bending at the ends of the circular arcs is not possible.

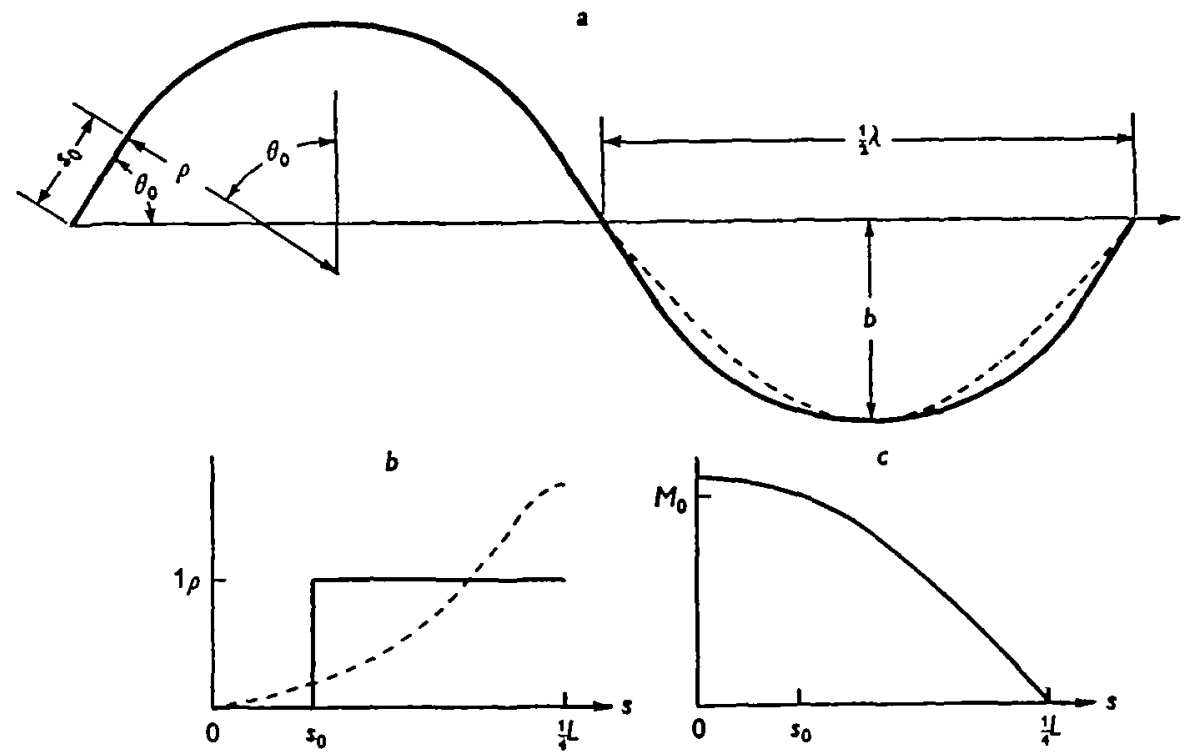

Fig. I. (a) Waveform constructed of circular arcs and straight lines, with $\theta_{0}=1$ radian and $s_{0}=0.4 \rho$. In the right half the dashed line represents a sinusoidal waveform with the same amplitude and wavelength. (b) Variation in curvature along the flagellum for the first quadrant of the waveform in (a) (colid lines) and for the corresponding quadrant of a sine wave (dashed lines). (c) Variation in viscous bending moment, $M(s)$, along the flagellum for the first quadrant of the waveform in $(a)$.

The normal wave pattern appears to be composed of circular arcs separated by shorter straight regions. A symmetrical wave pattern of this type, with proportions comparable to the wave patterns in the photographs, is illustrated in Text-fig. I $a$, and compared with a sinusoidal wave pattern. This wave pattern involves an abrupt change in curvature along the flagellum, and therefore an abrupt bending and unbending as the wave-pattern moves along the flagellum, as illustrated in Text-fig. I $b$. The actual rate of change of curvature, or rate of bending, cannot be measured accurately on these photographs, but in many cases the transition between straight and bent regions appears to be complete within a length of $1-2 \mu$, which would mean that the transition between straight and bent states requires no more than $2 \mathrm{msec}$. 
Text-fig. I $a$ also illustrates the parameters which may be used to describe the wave. Three parameters (in addition to frequency) are required to describe this type of wave, in contrast to a sinusoidal wave which can be described by two parameters.

Average values of wave parameters obtained for spermatozoa of the three species are listed in Table r. Stroboscopic measurements on at least 100 spermatozoa were averaged to obtain the beat frequency, $f$, which is the frequency at which waves originate at the head end of the sperm flagellum. The other values represent averages of measurements on photographs of ten or more spermatozoa. $\rho, \lambda$ and $L$ are the

Table I. Parameters of the bending waves

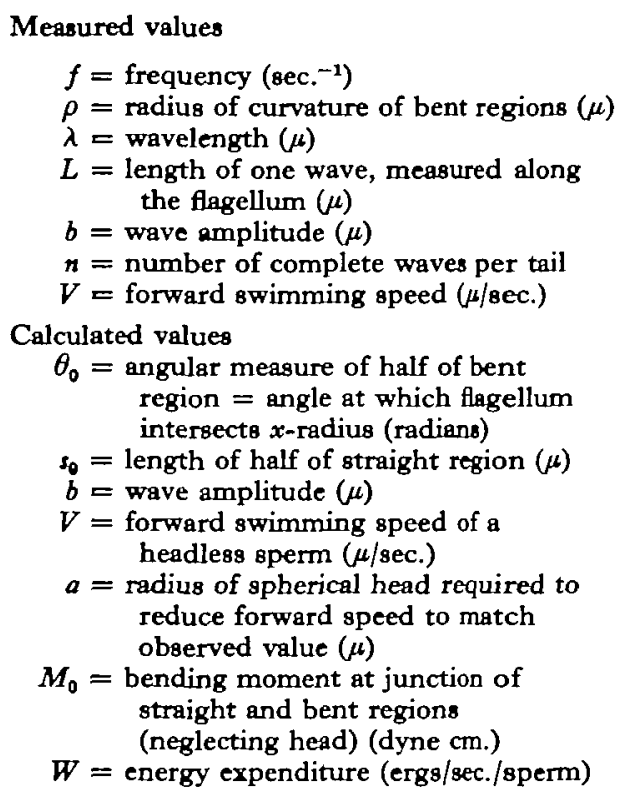

\begin{tabular}{|c|c|c|}
\hline Lytechinus & Ciona & Chaetopterus \\
\hline 30 & 35 & $26 \cdot 5$ \\
\hline 5.05 & $5 \cdot 5$ & $4 \cdot 3$ \\
\hline $22 \cdot 6$ & 22 & 19.5 \\
\hline $29 \cdot 6$ & 30 & 25.5 \\
\hline $4 \cdot 6$ & $4 \cdot 3$ & 3.8 \\
\hline $\mathrm{I} \cdot 4$ & $I \cdot 3$ & $1 \cdot 25$ \\
\hline $15^{\top}$ & 165 & 105 \\
\hline I.O & $I \cdot 13$ & 0.93 \\
\hline 2.6 & $1 \cdot 25$ & 2.4 \\
\hline 4.5 & 4.3 & $3 \cdot 6$ \\
\hline 192 & $230^{\circ}$ & 145 \\
\hline 0.75 & $1 \cdot 4$ & 1.0 \\
\hline $2.9 \times 10^{-10}$ & $4.0 \times 10^{-10}$ & $1 \cdot 6 \times 10^{-10}$ \\
\hline $3.1 \times 10^{-7}$ & $4.3 \times 10^{-7}$ & $1 \cdot 3 \times 10^{-7}$ \\
\hline
\end{tabular}

easiest wave parameters to measure accurately, but $\rho$, $s_{0}$, and $\theta_{0}$ are a more convenient set for mathematical analysis of the wave movement. The wave parameters are interrelated by the following equations:

$$
\begin{aligned}
L & =4\left(s_{0}+\rho \theta_{0}\right), \\
\lambda & =4\left(s_{0} \cos \theta_{0}+\rho \sin \theta_{0}\right), \\
b & =s_{0} \sin \theta_{0}+\rho\left(\mathrm{I}-\cos \theta_{0}\right) .
\end{aligned}
$$

Values of $b$ have been calculated by equation (3) for comparison with the observed values to give an indication of the accuracy of the measurements.

Ciona spermatozoa frequently remained motile, with the normal type of wave pattern illustrated in Pl. I, figs. 3 and 4 , for more than 30 min. on a slide. On the other hand Lytechinus spermatozoa showed this normal type of wave pattern for only a short time after being placed on the microscope slide. After a few minutes, when the motility began to decrease, wave patterns with more nearly sinusoidal forms were found, as illustrated in Pl. I, fig. 8. The period of normal motility of Lytechinus spermatozoa could be extended by further additions of organic compounds (e.g., $0 \cdot$ I $M$ urea) to the sea-water solution. 


\section{Behaviour near the ends of the flagellum}

The proximal portion of the flagellum, adjacent to the head, is often found to be bent into a circular arc with a smaller radius than that characteristic of the waves farther along the flagellum. Some additional examples are shown in Pl. I, figs. 9-I r. The development of bending waves in this region is illustrated by the photographs in Pl. I, fig. II, and Pl. 2, figs. I and 2. Pl. I, fig. II, was obtained by multiple flashes at a frequency slightly greater than the beat frequency; Pl. 2, figs. I and 2, show headless spermatozoa moving at reduced speed. After the initial bending, involving a short region with half or less the normal radius of curvature, the radius of curvature increases as the bent region extends along the flagellum. As a result of this behaviour the amplitude of oscillation of the head of the spermatozoon from side to side as it swims is significantly less than the amplitude of the flagellar wave pattern. Although this behaviour may be advantageous in the sense of reducing the power required for moving the head from side to side through the viscous medium, it cannot be a simple result of the resistance to movement provided by the head, since headless spermatozoa also show bends of increased curvature near the anterior end of the flagellum.

The envelope of beat of the flagellum of a normal spermatozoon in good condition extends with nearly constant amplitude to the distal end of the flagellum, as illustrated by Gray (1955). Photographs (Pl. I, figs, 2,6) and stroboscopic observations also show that bending occurs normally up to the end of the flagellum. After the initial decrease in curvature near the anterior end the curvature of the bends remains approximately constant as they move along the flagellum, although this is not shown as clearly as in the longer flagellum of Ceratium (Brokaw \& Wright, I963). When a bent region reaches the distal end it becomes gradually shorter, but it continues to form a circular arc with no change in its radius of curvature. In other words the elements of the flagellum near the distal end behave exactly as they would be expected to if the flagellum continued; proximity to the end does not appear to influence their behaviour. This is true in spite of the probability that the distribution of bending moment on the flagellum will change near the end of the flagellum.

When a distal portion of the flagellum was broken off, the movement near the new distal end differed significantly from that normally observed near the distal end of the flagellum. The envelope of beat displayed a pronounced node a few microns from the distal end, and resembled that typically found with ATP-reactivated, glycerinated flagella of Polytoma uvella (Brokaw, 1962, I963). Photographs and stroboscopic observations did not show short bent regions of normal radius near the distal end; instead the flagellum appeared to straighten quickly soon after a bent region reached the end (Pl. 2, fig. 3).

The special properties of the normal end of the flagellum which differentiate its behaviour from that of a broken end are not indicated, since breaking the flagellum might cause various types of damage. However, these properties may be related to the presence of the thinner terminal piece of the flagellum, which is only rarely seen in the photographs but can be seen by dark-field or electron microscopy and has a modified ultrastructure (Afzelius, 1955, I959). 


\section{Variant wave patterns}

Bending waves with greatly reduced radii of curvature were observed when Chaetopterus spermatozoa were suspended in sea-water solutions to which methyl cellulose had been added to increase the viscosity. The details of response to increased viscosity will not be discussed quantitatively in this paper, but some examples of this type of wave pattern are illustrated in $\mathrm{Pl}$. 2, figs. 4 and 5. These highly curved wave patterns are stable, reproducible, planar waves which propel the spermatozoa effectively in very viscous solutions. The configuration of the wave resembles that found normally on the flagellum of spermatozoa of the urodele, Cryptobranchus alleganiensis (Baker, 1963). Since the radius of curvature of bending near the head of the spermatozoon is not decreased in proportion to the decrease in curvature in the major portion of the wave, increased viscosity appears to act in part by limiting the increase in radius of curvature which normally occurs as a bend develops and leaves the proximal end of the flagellum. The same type of movement in viscous solutions was found occasionally with headless spermatozoa, and with ATP-reactivated, glycerinated spermatozoa, under conditions similar to those for Polytoma flagella (Brokaw, 1963). It has also been observed with spermatozoa of Ciona or Lytechinus, but only under more restricted conditions which have not yet been fully characterized.

The ability of a flagellum to bend at a smaller than normal radius of curvature is also illustrated by the wave patterns observed on spermatozoa which have become attached to the slide or coverglass by their heads. As shown in Pl. 2, figs. 6-Io, the radius of curvature is strongly reduced near the head of the spermatozoon, and increases towards a normal value as the waves move along the flagellum. In addition, the beat frequency of these spermatozoa is usually Io- $30 \%$ less than that of freely swimming spermatozoa. This effect also has been observed consistently with spermatozoa of Ciona.

The bent regions in wave patterns of attached spermatozoa often are not exactly circular arcs. The curvature appears to be slightly greater in the proximal portion of a bent region than in the distal portion. This asymmetry can be detected by attempting to fit the bends with circular arcs, but the variation in curvature within a bend is too small for accurate measurement.

One of the effects of thiourea on sperm movement (Brokaw, 1964) is a selective inhibition of the distal portion of the flagellum, as illustrated in Pl. 2, fig. Ir. There appears to be a sharp transition between the actively bending region and the inhibited region, which appears to be very stiff and shows no indication of passive wave propagation. When the distal portion is inhibited in this manner the orientation of the head of the spermatozoon does not oscillate as it does during normal swimming movements. The head maintains a constant orientation relative to the straight distal region, even if this requires a decrease in wavelength or an increase in curvature in the actively bending region of the flagellum. This effect has been found with Ciona and Lytechinus spermatozoa, at thiourea concentrations of $0 . \mathrm{I}-0.2 \mathrm{M}$, but has not been found with Chaetopterus spermatozoa, which appear to be much less permeable to thiourea. Since the distal inhibition is not immediate, and is not found with ATP-reactivated, glycerinated spermatozoa, it may result from an inhibition of metabolic reactions (Dubois \& Erway, 1946) which produce ATP. If these reactions are localized near 
the proximal end of the flagellum the production of ATP may not be sufficient to allow it to reach the distal portion of the flagellum.

The variant wave patterns described so far can be reproducibly obtained under appropriate conditions. In addition photographs have been obtained of variant wave patterns which apparently represent aberrant behaviour of individual spermatozoa. These are an additional potential source of information about the wave-generating capabilities of flagella, but cannot be studied in detail until they can be obtained consistently. Three examples of this type of wave-pattern variation are shown in Pl. 2, figs, 12-I4. Pl. 2, fig. I2 illustrates a transient aberration; the abnormal wave patterns shown by the first two flashes are followed by two nearly normal patterns. In contrast to this the example in Pl. 2, fig. I4 shows an aberration which was repeated consistently for at least two full beat cycles.

These three photographs show that deviations from the normal wave pattern can include patterns in which the bent region does not maintain the configuration of a circular arc. An important question which might be answered by further study of aberrations in the wave pattern is the following: Is a local aberration in the wave pattern propagated at the same rate as the wave, or does it remain at a point on the flagellum? On the basis of a very few observations it appears that both of these two types of behaviour may be possible; figs. $\mathrm{I}_{3}$ and $\mathrm{I}_{4}$ in Pl. 2 may represent these two types of behaviour.

\section{MATHEMATICAL ANALYSIS}

This analysis of movement by waves of the type shown in Text-fig. $\mathrm{I} a$ is based on the method used by Gray \& Hancock (1955) to analyse movement by sinusoidal waves. The analysis assumes that the force on any element, $d s$, along the length of the flagellum as it moves against the viscous resistance of the medium is the same as that which would be experienced by a similar element of a long, straight filament moving rectilinearly with the same velocity. The movement of an element, $d s$, is most easily specified in terms of $V_{s}$, the phase velocity of the wave along the flagellum, with respect to points on the flagellum $\left(V_{s}=f L\right)$, and $V_{e}$, the phase velocity of the wave along the $x$ axis (Text-fig. $1 a$ ) with respect to a co-ordinate system fixed in the viscous medium. The wave velocity, $V_{w}$, used by Gray \& Hancock (1955) is equal to $V_{s} \lambda / L$, and the average forward swimming speed, $V$, is related to these parameters by the following equation:

$$
V=V_{w}-V_{e}=(\lambda / L) V_{s}-V_{e}
$$

In terms of $V_{s}$ and $V_{e}$, the tangential and normal components of the force acting on an element, $d s$, indicated in Text-fig. $2 a$ are given by

$$
\begin{aligned}
d F_{L} & =C_{L}\left(V_{s}-V_{e} \cos \theta\right) d s, \\
d F_{N} & =C_{N} V_{e} \sin \theta d s,
\end{aligned}
$$

where $\theta$ is the angle of inclination between the $x$ axis and the element, $d s$, and $C_{L}$ and $C_{N}$ are, respectively, the tangential and normal drag coefficients. The analysis assumes that the drag coefficients are independent of velocity, although Machin (1958) has indicated that this may not be strictly true. 

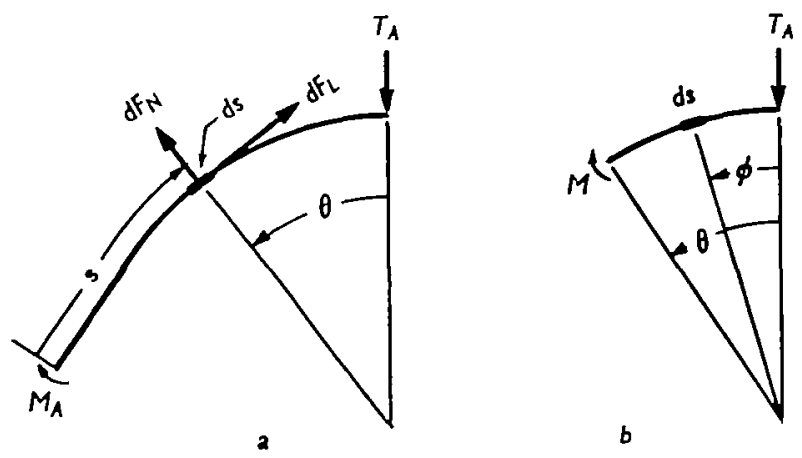

Fig. 2. Locations of bending moments and forces.

\section{Forward swimming speed}

The forward thrust along the $x$ axis resulting from the movement of any element, $d s$, is

$$
-d F_{x}=-d F_{L} \cos \theta+d F_{N} \sin \theta .
$$

The forward thrust generated by a complete wave is obtained by integrating equation (6) over one quadrant of the wave, and multiplying by 4 . The integrals turn out to be much more easily handled than those involved in the analysis of sinusoidal wave movement. For the straight region

$$
\int_{s=0}^{s-s_{\bullet}}-d F_{x}=s_{0}\left(C_{N} V_{e} \sin ^{2} \theta+C_{L} V_{e} \cos ^{2} \theta-C_{L} V_{s} \cos \theta\right) .
$$

For the curved region

$$
\int_{s=\varepsilon_{v}}^{s-s_{0}+\rho \theta_{0}}-d F_{x}=\frac{1}{2} \rho\left[C_{N} V_{e}\left(\theta_{0}-\sin \theta_{0} \cos \theta_{0}\right)+C_{L} V_{e}\left(\theta_{0}+\sin \theta_{0} \cos \theta_{0}\right)-2 C_{L} V_{s} \sin \theta_{0}\right] .
$$

In the absence of a head, or any other added resistances to forward movement, the total forward thrust of a complete wave must be $o$, and equations (8) and (9) lead to

$$
\frac{V_{e}}{V_{s}}=\frac{\lambda C_{L}}{L C_{N}+\left(C_{N}-C_{L}\right)\left(2 \rho \sin \theta_{0} \cos \theta_{0}-2 \rho \theta_{0}-\lambda \cos \theta_{0}\right)} .
$$

To check the validity of this analysis, measurements were made on the headless spermatozoa in Pl. 2, figs. I and 2, and three other photographs, and used to calculate the value of $C_{N} / C_{L}$. The results are given in Table 2. This is not an exact method, since the flagellum does not contain an integral number of complete waves and the wave parameters vary along the flagellum, particularly near the anterior end. The calculated values of $C_{N} / C_{L}$ agree reasonably well with the theoretical value of 2 for a very thin filament (Hancock, 1953) and validate the hydrodynamic assumptions on which this analysis is based.

The above derivation may be more exact than that based on sinusoidal waves, but the swimming velocities given by equations (4) and (I0) differ only slightly from those calculated by equation xxi of Gray \& Hancock (1955). For example, for the 
Table 2. Movement of headless spermatozoa of Lytechinus

\begin{tabular}{|c|c|c|c|c|c|}
\hline \multirow[b]{2}{*}{ Measured values } & \multicolumn{5}{|c|}{ Photograph no. } \\
\hline & 10 & $11 / 12$ & I9 & $\begin{array}{c}28 / 29 \\
\text { (Pl. 2, fig. r) }\end{array}$ & (Pl. 2, fig. 2) \\
\hline$\rho(\mu)$ & $5 \cdot 6$ & $4 \cdot 9$ & 4.7 & 5.5 & 4.9 \\
\hline$\lambda(\mu)$ & 22 & $2 \mathrm{I}$ & 20 & 23 & 21 \\
\hline$L(\mu)$ & 27 & 27 & $25^{\circ} 4$ & $32 \cdot 5$ & $28 \cdot 5$ \\
\hline$\theta(\mathrm{rad})$. & 0.87 & 0.98 & 0.95 & $1 \cdot 18$ & 1.07 \\
\hline $\begin{array}{c}V_{\cdot} / V_{s} \\
\text { Calculated values }\end{array}$ & 0.60 & 0.57 & 0.63 & 0.50 & 0.52 \\
\hline$C_{N} / C_{L}$ & $2 \cdot 13$ & $x \cdot 95$ & $x \cdot 69$ & $1 \cdot 93$ & I.99 \\
\hline
\end{tabular}

waveform in Text-fig. I $a$, the equation of Gray \& Hancock gives a value which is $2 \%$ greater than the value given by equations (4) and (10).

The average forward swimming speed of a spermatozoon with a head can be estimated by assuming that the resistance of the head is balanced by the forward thrust generated by a complete wave, times $n$, the number of waves on the flagellum. If the resistance of the head is expressed as $V C_{H}$, where $C_{H}$ is a drag coefficient for the head, and $C_{N}={ }_{2} C_{L}$, the swimming speed becomes

$$
V=\frac{\lambda}{L} V_{s}\left(\mathrm{x}-\frac{\mathrm{I}+C_{H} / n L C_{L}}{2+C_{H} / n L C_{L}+(\mathrm{I} / L)\left(2 \rho \sin \theta_{0} \cos \theta_{0}-2 \rho \theta_{0}-\lambda \cos \theta_{0}\right)}\right) .
$$

$C_{I}$ may be estimated by assuming that the head acts as a sphere with an effective radius $a$, so that Stokes's law gives

$$
C_{H}=6 \pi \eta a,
$$

where $\eta$ is the viscosity of the medium. $C_{L}$ was determined by Hancock (1953) and Gray \& Hancock (1955) to be given by

$$
C_{L}=\frac{2 \pi \eta}{\log (2 \lambda / d)-0.5}
$$

where $d$ is the radius of the flagellum, approximately $0 \cdot 1 \mu$.

Using equations (I2) and (13), and an average value of $20 \mu$ for $\lambda$,

$$
C_{H} / C_{L}=16 \cdot 5 a \text {. }
$$

Equations (II) and (I4) were used to calculate values of $a$, given in Table I, which would give a calculated swimming speed equal to the observed swimming speed of the spermatozoa. The values are in the right range, but are not consistent with the impression that Ciona spermatozoa have the smallest heads. However, this estimate of head size will be very sensitive to errors in the measurement of the swimming speed and the wave parameters, since the resistance of the head is a small part of the total resistance of the spermatozoon.

\section{Bending moment}

Machin (1958) gave an expression for the sinusoidal variation of the bending moment required to oppose viscous forces on a flagellum moving with sinusoidal 
waves of small amplitude. For waves composed of circular arcs and straight lines the variation in bending moment along the length can be obtained without restriction to waves of small amplitude. Only the most idealized case, in which the effect of the head, and other end effects, are neglected, will be treated.

The bending moment will vary periodically along the length of the wave, and will have its maximum absolute values, $M_{A}$, at the points where the wave crosses the $x$ axis, and will be $O$ at the crests of the waves. There will also be shearing forces in the flagellum which will have maximum values, $T_{A}$, at the wave crests and will be $\circ$ at the points where the wave crosses the $x$ axis. These are indicated for one quadrant of the wave in Text-fig. $2 a$. In the straight region of the wave, the bending moment, $M$, at any point $s$ (as defined in Text-fig. $2 a$ ) can be obtained in the usual manner for a uniformly loaded straight beam:

$$
M=M_{A}-\frac{1}{2} s^{2} C_{N} V_{e} \sin \theta_{0} .
$$

In the bent region of the wave, $M$ is obtained by integration of the moments due to the forces on each element, $d s$, in the circular segment illustrated in Text-fig. $2 b$ and adding the moment due to $T_{A}$ :

$$
\begin{array}{r}
M=\rho \sin \theta T_{A}+\int \rho[\mathrm{I}-\cos (\theta-\phi)] d F_{L}-\rho \sin (\theta-\phi) d F_{N}, \\
=\rho \sin \theta T_{\Delta}+\int_{0}^{\theta} \rho^{2} C_{L}[\mathrm{I}-\cos (\theta-\phi)]\left(V_{s}-V_{e} \cos \phi\right) d \phi \\
-\int_{0}^{\theta} \rho^{2} C_{N} V_{e}(\sin (\theta-\phi) \sin \phi d \phi), \\
=\left\{\rho T_{\Delta}-\frac{1}{2}\left[\rho^{2} V_{e}\left(C_{N}+C_{L}\right)\right]-\rho^{2} C_{L} V_{s}\right\} \sin \theta+\rho^{2} C_{L} V_{s} \theta \\
+\frac{1}{2}\left[\rho^{2} V_{e}\left(C_{N}+C_{L}\right)\right] \theta \cos \theta .
\end{array}
$$

$T_{A}$ can be obtained by integration of the vertical components of the forces on each element, $d s$, for one quadrant of the wave

$$
\begin{aligned}
T_{\boldsymbol{A}} & =\int d F_{L} \sin \theta+d F_{N} \cos \theta, \\
& =\int_{0}^{t L} C_{L} V_{s} \sin \theta d s+\left(C_{N}-C_{L}\right) V_{e} \sin \theta \cos \theta d s, \\
& =C_{L} V_{s}\left[s_{0} \sin \theta_{0}+\rho\left(\mathrm{x}-\cos \theta_{0}\right)\right]+\left(C_{N}-C_{L}\right) V_{e}\left(s_{0} \sin \theta_{0} \cos \theta_{0}+\frac{1}{2} \rho \sin ^{2} \theta_{0}\right) .
\end{aligned}
$$

Equations ( 18 ) and (2I) can be combined to obtain the bending moment in the bent region at any point specified by the angle $\theta$. The bending moment, $M_{0}$, at the bending point, where $\theta=\theta_{0}$, can be simplified by using equation (ro) to give

$$
M_{0}=\frac{C_{L} \rho L V_{s}}{4}\left(1-\frac{\lambda V_{e}}{L V_{s}}\right) .
$$

'This relationship, which does not depend on the value of $C_{N} / C_{L}$, can also be obtained by more direct arguments.

Since $M_{0}$ is also given by equation (I5) when $s=s_{0}, M_{A}$ can be found in terms of $M_{0}$, to give the desired expression for $M$ in the straight region of the flagellum:

$$
M=M_{0}+\frac{1}{2}\left(s_{0}^{2}-s^{2}\right) C_{N} V_{e} \sin \theta_{0} .
$$


Equations (18) and (23) are illustrated by the curve of $M$ against $s$ in Text-fig. I $c$. The bending moment varies almost sinsusoidally; in fact, if it did vary sinusoidally, the resulting wave form might not be detectably different from that in Text-fig. I $a$.

\section{Energy expenditure}

The flagellum can perform external work only when it is bending and unbending at the junctions between straight and bent regions, where the bending moment required to overcome the viscous resistance of the medium is $M_{0}$, given by equation (22). The rate at which work is done against the viscous resistance of the medium at the bending point is therefore:

$$
W_{0}=M_{0} \frac{d \theta}{d t}=\frac{M_{0} V_{s}}{\rho} .
$$

The rate at which work is done by the whole flagellum is obtained by multiplying by the number of bending and unbending points, which is $4^{n}$ :

$$
W=4 n \frac{M_{0} V_{s}}{\rho}=n L C_{L} V_{s}^{2}\left(\mathrm{I}-\frac{\lambda V_{e}}{L V_{s}}\right)
$$

The energy expenditures given by equation (25) are similar to those estimated by Carlson (1959) for sinusoidal wave movement. For example, for the wave pattern in Text-fig. I $a$, the final term in equation (25) has the value of 0.59 , and the corresponding term in the equation given by Carlson has the value 0.61 . The equation given by Rothschild ( 1963 ) gives lower values for the energy expenditure.

The energy expended in moving the head of the spermatozoon forward and from side to side through the medium will probably be about $5 \%$ of the total energy expenditure. Although not explicitly incorporated in equation (25), this portion of the energy expenditure can be included by using the calculated values of $V_{e}$ for a headless spermatozoon (equation ro) rather than the observed values for spermatozoa with heads. The energy expenditures given in Table I were obtained in this way.

\section{DISCUSSION}

The observations on the spermatozoa of the three species with which the present paper is concerned, together with the earlier observations (Brokaw \& Wright, 1963) and unpublished observations on spermatozoa of other marine invertebrates, all indicate that the general form of flagellar bending waves is not that of a sine wave, but is instead composed of circular arcs of constant bending separated by short unbent regions. This distinction between two very similar waveforms has been made possible by the higher photographic resolution now available. It simplifies the mathematical analysis of the wave movement, but has little effect on the estimates obtained for forward velocity and energy expenditure. The new photographs fully agree with the most important conclusion of earlier work (Gray, 1955), namely that the maintenance of full wave amplitude as the bending waves pass along a flagellum requires active bending all along the flagellum.

The major significance of the new waveform relates to the manner in which active bending is controlled as bending waves pass along the flagellum. The constancy of curvature within a bent region indicates that the local curvature is independent of the 
immediate, local value of the bending moment imposed upon the moving flagellum by viscous forces. This bending moment varies greatly within a bent region, as indicated in Text-fig. I c. It will tend to decrease the curvature in the distal part of a bent region and increase the curvature in the proximal part of a bent region, giving an effect similar to the damping of passively propagated bending waves (Machin, 1959). A slight effect of this nature, which may have a different explanation, has been noticed with attached spermatozoa (P1. 2, figs. 6-10), but freely swimming spermatozoa only occasionally show any indication of this effect.

To maintain constant curvature each element along the length of the flagellum must generate a bending moment equal to that imposed by viscous forces. This opposing moment may be generated actively or passively; in either case the flagellum has a high resistance to bending out of its normal waveform; it is effectively very stiff. Regardless of its mechanism this high resistance to bending must be greatly reduced at the junctions between straight and bent regions, where rapid bending and unbending occurs. The work required to bend and unbend at these transition points against the resistance to bending maintained in the other regions would be prohibitively great. The high resistance to bending cannot be attributed to the presence of a stiff, inert supporting structure in parallel with the structures responsible for active bending and unbending, and is probably a property of the same structures which are responsible for bending and unbending.

The presence of circular arcs in flagellar bending waves was initially interpreted as evidence that the active bending elements of the flagellum have non-linear properties which cause them to operate in an 'on-or-off' manner, giving a fairly abrupt transition between straight and bent states in response to some activating stimulus (Brokaw \& Wright, 1963). After bending, the bent elements must become stiff and maintain their bend until they abruptly unbend and return to a stiff, straight state. This transition is indicated by the solid curve in Text-fig. $I b$. The control of bending which would be required to produce sinusoidal bending waves would appear to be much more complicated, as indicated by the dashed curve in this figure.

This interpretation must now be modified to take into account the alteration of the radius of curvature of the bent regions which can occur in response to environmental conditions, such as the viscosity of the medium. The radius of curvature is determined by the amount of bending which occurs during the transition from the straight stiff state to the bent stiff state, and this can apparently be influenced by environmental conditions. Uniform bending - giving circular arcs-indicates a uniform response to uniform conditions.

In spermatozoa which are attached to the slide by their heads the bending is not uniform but decreases gradually towards the distal end of the flagellum. Consequently, if the local amount of bend remains constant between the times of bending and unbending, the curvature within a given bend, at any time, must decrease towards the distal end of the flagellum. A similar variation in curvature within a bent region should occur in any other situations where the curvature changes as the bending point progresses, such as normally occurs near the head of a spermatozoon. However, this variation in curvature within a bent region will be small and almost impossible to measure, since the rate of change of curvature along the length is small in the examples described here. A suggestion of this type of variation in curvature appears 
in many of the photographs of attached spermatozoa and a few of the photographs of bends near the head of swimming spermatozoa, but a confident statement that these bends deviate from circular arcs to the expected extent is not possible. A more definite answer might be obtained if conditions could be found which would cause an abrupt change in curvature at a point along the flagellum.

If the bends were always exactly circular arcs even when the curvature changed as the wave progressed, the constancy of bending probably could not be explained solely in terms of the response of localized bending elements. A continuing, internal interaction between the bending elements within a bent region would be required to maintain constant curvature. Such a hypothesis would be a radical departure from the idea of autonomous local bending elements which is basic to Machin's model (1958) for wave propagation, and its functional value to the spermatozoon is not obvious. On the other hand, the possibility of internal interaction between the bending elements is suggested by a few other observations, such as the behaviour of thioureainhibited spermatozoa ( $\mathrm{Pl}$. 2, fig. I I), where the head maintains a constant orientation with reference to the inhibited distal regions. This behaviour means that there is no net bending to either side as a result of activity in the uninhibited region; it might be quite simply explained by the presence in the structure of the flagellum of flexible, inextensible fibrils which are tied together at the head end of the flagellum and in the thiourea-inhibited region but are free to move relative to one another in the actively bending region. This model is consistent with the electron-microscope observations of Satir (1964), which suggest that the outer filaments of the ciliary axoneme do not change their lengths when the cilium bends.

However, the interaction involved in this particular type of simple model does not appear to provide any better explanation of the maintenance of constant curvatur = within a bent region. Until more conclusive evidence can be obtained the assumption of autonomous local bending elements, activated in an 'on-or-off' manner but with the amount of bending determined by environmental conditions, appears to be a satisfactory working hypothesis.

\section{SUMMARY}

I. Photomicrographs giving greater resolution than previously available have been obtained for the spermatozoa of a sea urchin, a tunicate, and an annelid.

2. The bending waves are not sine waves. They contain circular arcs, in which the amount of bending is constant, separated by shorter regions which are nearly straight.

3. The flagellum must be effectively very stiff to maintain this waveform as it moves through the medium, but this stiffness must be greatly reduced at the junctions between straight and circular regions, where the flagellum is actively bending and unbending.

4. This type of wave pattern may be generated by a simple 'on-or-off' activation of bending elements, but the amount of bending which occurs can be influenced by environmental conditions, such as the viscosity of the medium.

5. A mathematical analysis of the wave movement can be carried out more easily than with sinusoidal waves, and expressions have been obtained for the forward swimming speed, the distribution of bending moment along the flagellum, and the energy expenditure for movement, in terms of the parameters of the waves. 
6. The expression for the forward swimming speed accurately predicts the swimming speed of headless spermatozoa, confirming the validity of the hydrodynamic assumptions used in this analysis.

\section{REFERENCES}

AfzELius, B. A. (1955). The fine structure of the sea-urchin spermatozoon as revealed by the electron microscope. Z. Zellforsch. 42, $134-48$.

Afzelius, B. A. (1959). Electron microscopy of the sperm tail. Results obtained with a new fixative. 7. Biophys. Biochem. Cytol. 5, 269-78.

BAKER, C. L. (1963). Spermatozoa and spermateleosis in Cryptobranchus and Necturus. F. Tennessee Acad. Sci. 38, 1-1 I.

Brokaw, C. J. (1962). Studies on isolated flagella. In Spermatozoan Motility (Bishop, D. W., ed), pp. 269-78. American Association for the Advancement of Science.

Brokaw, C. J. (1963). Movement of the flagella of Polytoma trvella. F. exp. Biol. 40, 149-56.

ВroкAw, C. J. (1964). Thiourea-induced alterations of flagellar waveforms. 7 . Cell Biol. 23, I 5 A.

Brokaw, C. J. \& Wright, L. (1963). Bending waves of the posterior flagellum of Ceratium. Science, I42, I $169-70$.

Carison, F. D. (1959). The motile power of a swimming spermatozoon. Proc. First National Biophysics Conf. (Quastler, H. and Morowitz, H. J., eds.), pp. 443-9. Yale University Press.

DuBois, K. P. \& ERway, W. F. (1946). Studies on the mechanism of action of thiourea and related compounds. II. Inhibition of oxidative enzymes and oxidations catalysed by copper. 7 . Biol. Chem. I65, 7 I I-2I.

Gray, J. (1955). The movement of sea-urchin spermatozoa. F. Exp. Biol. 32, 775-801.

Gray, J. \& HaNcock, G. J. (1955). The propulsion of sea urchin spermatozoa. F. Exp. Biol. 32, 802-14.

Hancock, G. J. (1953). The self-propulsion of microscopic organisms through liquids. Proc. Roy. Soc A, 217, 96-121.

Machin, K. E. (r958). Wave propagation along flagella. F. Exp. Biol. 35, 796-806.

Machin, K. E. (I963). The control and synchronization of flagellar movement. Proc. Roy. Soc. B, $158,88-104$.

Pringle, J. W. S. (1957). Myogenic Rhythms. In Recent Advances in Invertebrate Physiology (Scheer, B. T., ed.), pp. 99-115. University of Oregon.

Rothschild, LoRD (I96I). Sperm energetics. An account of work in progress. In The Cell and the Organism (Ramsay, J. A. and Wigglesworth, V. B., eds.), pp. 9-21. Cambridge University Press.

ROTHSCHILD, LORD (1963). Non-random distribution of bull spermatozoa in a drop of sperm suspension. Nature, Lond., 198, $1221-2$.

SatiR, P. (1964). Filament-matrix interaction during ciliary movement: Inferences drawn from electron microscopy of the distal end of the ciliary shaft of lamellibranch gill cilia. $\mathcal{F}$. Cell Biol. 23, $82 \mathrm{~A}$.

TyLER, A. (1949). A simple, non-injurious, method for inducing repeated spawning of sea-urchins and sand dollars. The Collecting Net, 19, 19-20.

TYLER, A. (1953). Prolongation of life-span of sea urchin spermatozoa, and improvernent of the fertilization-reaction, by treatment of spermatozoa and eggs with metal-chelating agents (amino acids, versene, DEDTO, oxine, cupron). Biol. Bull., Woods Hole, ra4, 224-39.

\section{EXPLANATION OF PLATES}

Plate I

Fig8. I-6. 'Normal' wave patterns of spermatozoa. Flash rate, 50/sec. Figs. 1, 2. Spermatozoa of Lytechinus. Figs. 3, 4. Spermatozoa of Ciona. Figs. 5, 6. Spermatozoa of Chaetopterus.

Fig. 7. Enlargement (approx. $3 \cdot 6 \times$ ) of a portion of fig. I, with circular arcs and straight lines drawn on the image of the flagellum.

Fig. 8. Typical wave pattern of spermatozoa of Lytechinus after several minutes on the microscope slide. Flash rate, 5o/sec.

Figs. 9, Io. Spermatozoa of Chaetopterus, illustrating greater curvature of the flagellum near the head. Flash rate, 40/sec.

Fig. II. Spermatozoon of Ciona, illustrating the development of bending at the proximal end of the flagellum. Flash rate, $40 / \mathrm{sec}$. 

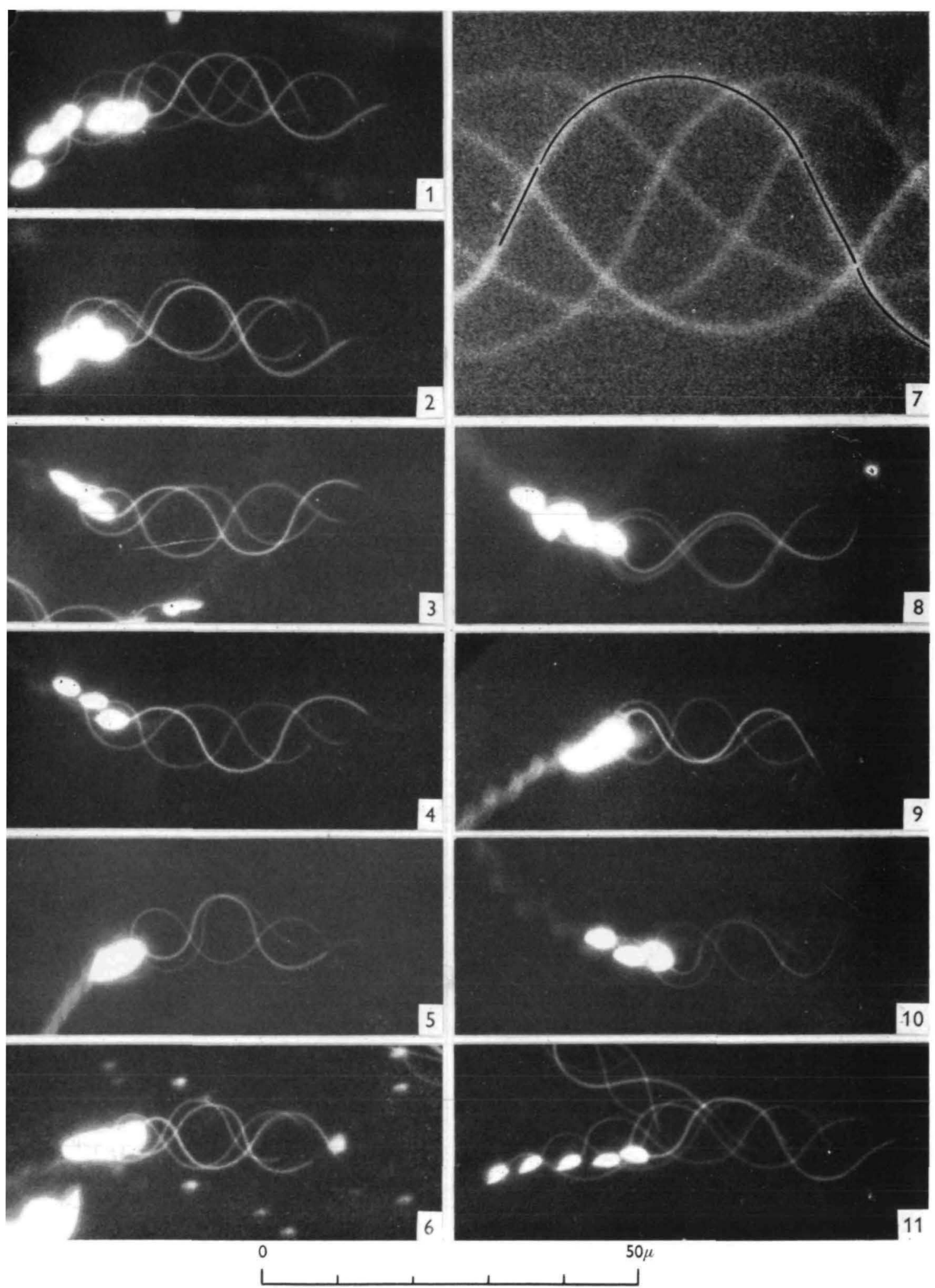

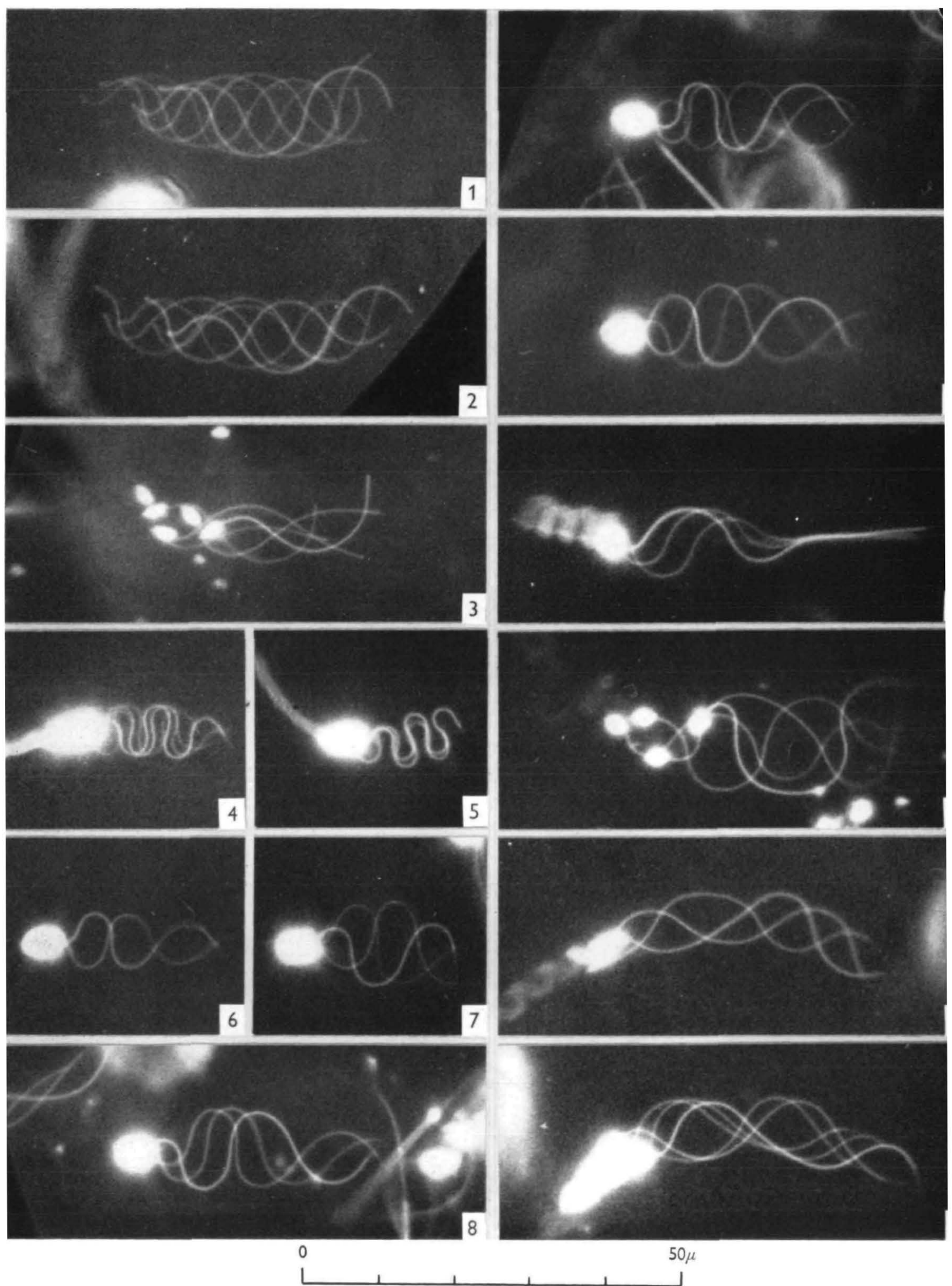


\section{Plate 2}

Figs. 1, 2. Headless spermatozoa of Lytechimus. Flash rate, 3o/sec.

Fig. 3. Broken spermatozoon of Ciona. Flash rate, $40 / \mathrm{sec}$.

Fig. 4. Spermatozoon of Chaetopterus in a solution having a viscosity of approx. 40 centipoise. Flash rate, 4 o/sec.

Fig. 5. Spermatozoon of Chaetopterus in a solution having a viscosity of approx. 300 centipoise. Flash rate, $20 / 8$ ec.

Figs. 6, 7. Wave patterns of Chaetopterus spermatozoa which are attached to the surface of the slide by their heads. Flash rate, $40 / \mathrm{sec}$.

Figs. 8-ro. Wave patterns of Lytechimus spermatozoa which are attached to the surface of the slide by their heads. Flash rate, 4o/sec.

Fig. I I. Spermatozoon of Ciona in a solution containing $0.175 \mathrm{M}$ thiourea, showing the inhibition of the distal end of the flagellum which occurs after several minutes in thiourea. Flash rate, $40 / \mathrm{sec}$.

Fig. I2. Aberrant wave-pattern of a spermatozoon of Ciona in a solution containing $0.175 \mathrm{M}$ thiourea. Flash rate, 3o/sec.

Fig. I3. Aberrant wave-pattern of a spermatozoon of Ciona in a solution having viscosity of approx. 5 centipoise. Flash rate, $40 / \mathrm{sec}$.

Fig. 14. Aberrant wave-pattern of a spermatozoon of Lytechinus. Flash rate, 50/8ec. 